\title{
Current status of antimicrobial prophylaxis during extracorporeal membrane oxygenation in Japan: a national survey
}

\author{
Yoshitaka Hara ${ }^{1} \cdot$ Tomoaki Yatabe $^{2}$ (1) $\cdot$ Koshiro Kikkawa $^{3} \cdot$ Tomoyuki Nakamura $^{1} \cdot$ Naohide Kuriyama $^{1}$. \\ Osamu Nishida ${ }^{1}$
}

Received: 6 July 2021 / Accepted: 12 August 2021 / Published online: 16 August 2021

(c) The Japanese Society for Artificial Organs 2021

\begin{abstract}
Infection during extracorporeal membrane oxygenation (ECMO) is a common complication that leads to increased mortality. Thus, antimicrobial prophylaxis during ECMO is often performed to prevent of nosocomial infections. However, the current status of antimicrobial prophylaxis during ECMO in Japan is unclear. Therefore, we conducted a national survey of members of the Japanese Society of Intensive Care Medicine (JSICM) to clarify the current status of antimicrobial prophylaxis during ECMO in intensive care units. An 11-question survey was devised to assess antimicrobial prophylaxis and surveillance practices during ECMO. A total of 253 hospitals responded. Of these, 235 hospitals were the JSICM-certified hospitals, and the response rate was $64 \%$. A total of 96 hospitals (39\%) administered antimicrobial prophylaxis during ECMO, and $17 \%$ of hospitals had a standardized protocol for antimicrobial prophylaxis during ECMO. Of these 96 hospitals, 79\% used single agents. First-generation cephalosporins were the most commonly used (54\%), followed by penicillins or penicillin-derived combinations (24\%), second-generation cephalosporins (7\%), and anti-methicillin-resistant Staphylococcus aureus agents (6\%). In conclusion, our survey revealed 39\% of hospitals administered antimicrobial prophylaxis during ECMO in Japan. First-generation cephalosporins were the agents most commonly used.
\end{abstract}

Keywords Antimicrobial prophylaxis $\cdot$ Extracorporeal membrane oxygenation $\cdot$ Survey

\section{Introduction}

Extracorporeal membrane oxygenation (ECMO) is a treatment for acute respiratory failure and has been of great interest during the COVID-19 pandemic. Infection during ECMO is a common complication that leads to increased mortality [1]. A previous review article described that about $20 \%$ of

Tomoaki Yatabe

yatabe.tomoaki@katch.ne.jp

$\triangle$ Osamu Nishida

nishida@fujita-hu.ac.jp

1 Department of Anesthesiology and Critical Care Medicine, Fujita Health University, 1-98, Dengakugakubo, Kutsukake-cho, Toyoake, Aichi 470-1192, Japan

2 Department of Anesthesiology and Intensive Care Medicine, Nishichita General Hospital, 3-1-1, Nakanoike, Tokai, Aichi 477-8522, Japan

3 Department of Anesthesiology, Ogaki Municipal Hospital, Ogaki, Gifu, Japan adult patients receiving ECMO acquired a culture-proven infection during ECMO [2]. Therefore, prevention of nosocomial infections during ECMO is important [2]. A previous survey demonstrated that $74 \%$ of ECMO centers administered antimicrobial prophylaxis [3]. However, antimicrobial exposure is associated with adverse consequences, including microbial drug resistance and Clostridioides difficile infections [4]. A previous review described that current evidence did not support routine prophylactic antimicrobial use in patients receiving ECMO support [2]. The latest Extracorporeal Life Support Organization (ELSO) guidelines for the management of adults undergoing ECMO did not mention antimicrobial prophylaxis [5]. The current status of antimicrobial prophylaxis during ECMO in Japan is unclear. Therefore, we conducted a national survey of members of the Japanese Society of Intensive Care Medicine (JSICM) to clarify the current status of antimicrobial prophylaxis during ECMO in intensive care units (ICUs). 


\section{Materials and methods}

This study was approved by the Fujita Health University Ethics Committee (HM20-235). The JSICM Clinical Trial Group also approved the study protocol (No. 38).

An 11-question survey was devised to assess antimicrobial prophylaxis and surveillance practices during ECMO. The questions pertained to items based on a previous study [3] and included the following: number of ICU beds, number of ECMO runs, infection surveillance using cultures, use of antimicrobial prophylaxis, and use of a standardized protocol for antimicrobial prophylaxis (full survey items are shown in Supplemental File 1). First, requests for participation were sent to members of the JSICM based on the mailing lists on February 24, 2021. Responses were collected from February 24 to March 10, 2021, in an electronic survey format using a commercial website (www.surveymonkey.com). Second, we sent an invitation letter on March 12, 2021 to the JSICM-certified hospitals that did not respond during the first time period. These responses were collected from March 15 to April 30, 2021, using an electronic survey system or by post.

Categorical data are presented as numbers (percentages), and continuous data as median (interquartile range).

\section{Results}

A total of 253 hospitals responded to the survey. Of these, 235 hospitals were JSICM-certified, and the response rate was $64 \%$. A summary of the survey responses is shown in the Table 1 . The median number of ICU beds was $10[8,14]$. Eighty-nine hospitals (35\%) performed ECMO on more than 6 cases per year, while 164 hospitals $(65 \%)$ performed fewer than 5 ECMO runs per year.

A total of 127 hospitals (51\%) performed routine surveillance. Most of the cultures were from samples obtained
Table 1 Responsiveness of each item

\begin{tabular}{|c|c|c|}
\hline Survey item & & $N=253$ \\
\hline Intensive care unit beds & $N$ & $10[8,14]$ \\
\hline \multicolumn{3}{|l|}{ ECMO runs per year } \\
\hline 0 & $N(\%)$ & $43(17)$ \\
\hline $1-5$ & $N(\%)$ & $121(48)$ \\
\hline $6-10$ & $N(\%)$ & $49(19)$ \\
\hline $11-$ & $N(\%)$ & $40(16)$ \\
\hline Routine surveillance cultures & & $N=251$ \\
\hline Yes & $N(\%)$ & $127(51)$ \\
\hline Blood & $N(\%)$ & $92(72)$ \\
\hline Sputum & $N(\%)$ & $109(86)$ \\
\hline Urine & $N(\%)$ & $68(54)$ \\
\hline How often do you perform routine surveillance cultures? & & $N=124$ \\
\hline Every $24 \mathrm{~h}$ & $N(\%)$ & $2(2)$ \\
\hline Every $48 \mathrm{~h}$ & $N(\%)$ & $11(9)$ \\
\hline Every $72 \mathrm{~h}$ & $N(\%)$ & $40(32)$ \\
\hline Other & $N(\%)$ & $71(57)$ \\
\hline Routine prophylaxis, Yes & $N(\%)$ & $96(39)$ \\
\hline $\begin{array}{l}\text { What percentage of ECMO patients are administrated antimicro- } \\
\text { bial prophylaxis? }\end{array}$ & $\%$ & $100[100,100]$ \\
\hline Standardized protocol, Yes & $N(\%)$ & $16(17)$ \\
\hline Monitor compliance, Yes & $N(\%)$ & $10(71)$ \\
\hline $\begin{array}{l}\text { Is the antimicrobial prophylaxis for ECMO patient single antimicro- } \\
\text { bial regimen? }\end{array}$ & $N(\%)$ & $71(79)$ \\
\hline \multicolumn{3}{|l|}{ Yes } \\
\hline \multicolumn{3}{|l|}{ Duration of prophylaxis } \\
\hline Pre-cannulation only & $N(\%)$ & $3(3)$ \\
\hline Pre-cannulation and for $24 \mathrm{~h}$ & $N(\%)$ & $8(9)$ \\
\hline Limited number of days & $N(\%)$ & $3(3)$ \\
\hline Duration of ECMO & $N(\%)$ & $33(37)$ \\
\hline Other/Don't know & $N(\%)$ & $42(47)$ \\
\hline
\end{tabular}

$N$ number, ECMO extracorporeal membrane oxygenation 
from the sputum ( $86 \%$ ), followed by those from the blood $(72 \%)$ and urine (54\%). The interval between of surveillance cultures varied, namely, every $24 \mathrm{~h}(2 \%)$, every $48 \mathrm{~h}(9 \%)$,

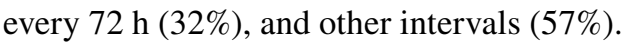

A total of 96 hospitals (39\%) administered antimicrobial prophylaxis during ECMO, and $17 \%$ of hospitals had a standardized protocol for antimicrobial prophylaxis. Of these 96 hospitals, $79 \%$ used single agents. First-generation cephalosporins were the most commonly used (54\%), followed by penicillins or penicillin-derived combinations (24\%), second-generation cephalosporins (7\%), anti-methicillin-resistant Staphylococcus aureus (MRSA) agents (6\%), carbapenems (4\%), third-generation cephalosporins (3\%), and echinocandin antifungal agents (1\%) (Fig. 1). Of the combined regimens, an anti-MRSA agent with a carbapenem was the most commonly used (82\%). Anti-MRSA agents were used in all combined regimens.

About half the hospitals (47\%) did not report the duration of antimicrobial prophylaxis. For the remainder of hospitals, duration of ECMO was the most common response.

\section{Discussion}

Our survey revealed that $39 \%$ of hospitals administered antimicrobial prophylaxis during ECMO in Japan. We believe that these results represent the current status in Japan to a certain extent, because $64 \%$ of the JSICM-certified hospitals responded. In addition, this survey revealed that only $16 \%$ of hospitals run ECMO $>10$ times per year, while a previous international survey reported that $82 \%$ of centers use ECMO $\geq 10$ times per year [1].

A 2010 ELSO survey of antimicrobial prophylaxis during ECMO reported that $74 \%$ of centers administered antimicrobial prophylaxis and $42 \%$ routinely administered antibacterial prophylaxis to all ECMO patients [3]. Forty-nine percent of the centers had a standardized protocol. Fewer than half the centers used a single agent, and first-generation cephalosporins were the most used in centers with a protocol, while vancomycin was the most used in those without a protocol. In centers using a combined regimen, none with a standardized protocol reported using carbapenems, whereas $14 \%$ of centers without a protocol reported using them. Routine infection surveillance was performed in $49 \%$ of the centers, and the cultures obtained were from blood (100\%) and sputum (48\%) samples. Another international survey performed in 2017 revealed that $55 \%$ of centers had a protocol for the use of prophylactic antibiotics and $61 \%$ had a protocol for infection surveillance, including cultures and inflammatory markers [1]. In our survey, $39 \%$ of hospitals administered antimicrobial prophylaxis and $79 \%$ of hospitals used single agents, unlike the results of previous surveys. On the other hand, only $17 \%$ of hospitals had a standardized protocol. Routine infection surveillance was performed in $51 \%$ of the centers, similar to the ELSO survey result. Despite the slow adoption of a standardized protocol in Japan, routine antimicrobial prophylaxis is not as popular as in other countries. As mentioned above, a previous review article and recent guidelines did not explicitly recommend routine antimicrobial prophylaxis. In addition, physicians
Fig. 1 Single-agent antimicrobial regimens. 1st First-generation cephalosporin, 2nd Secondgeneration cephalosporin, $3 r d$ Third-generation cephalosporin, Candi Echinocandin antifungal agent, Carba Carbapenem, MRSA Anti-MRSA agent, Penicillin Penicillin class, $P D C$ Penicillin-derived combination

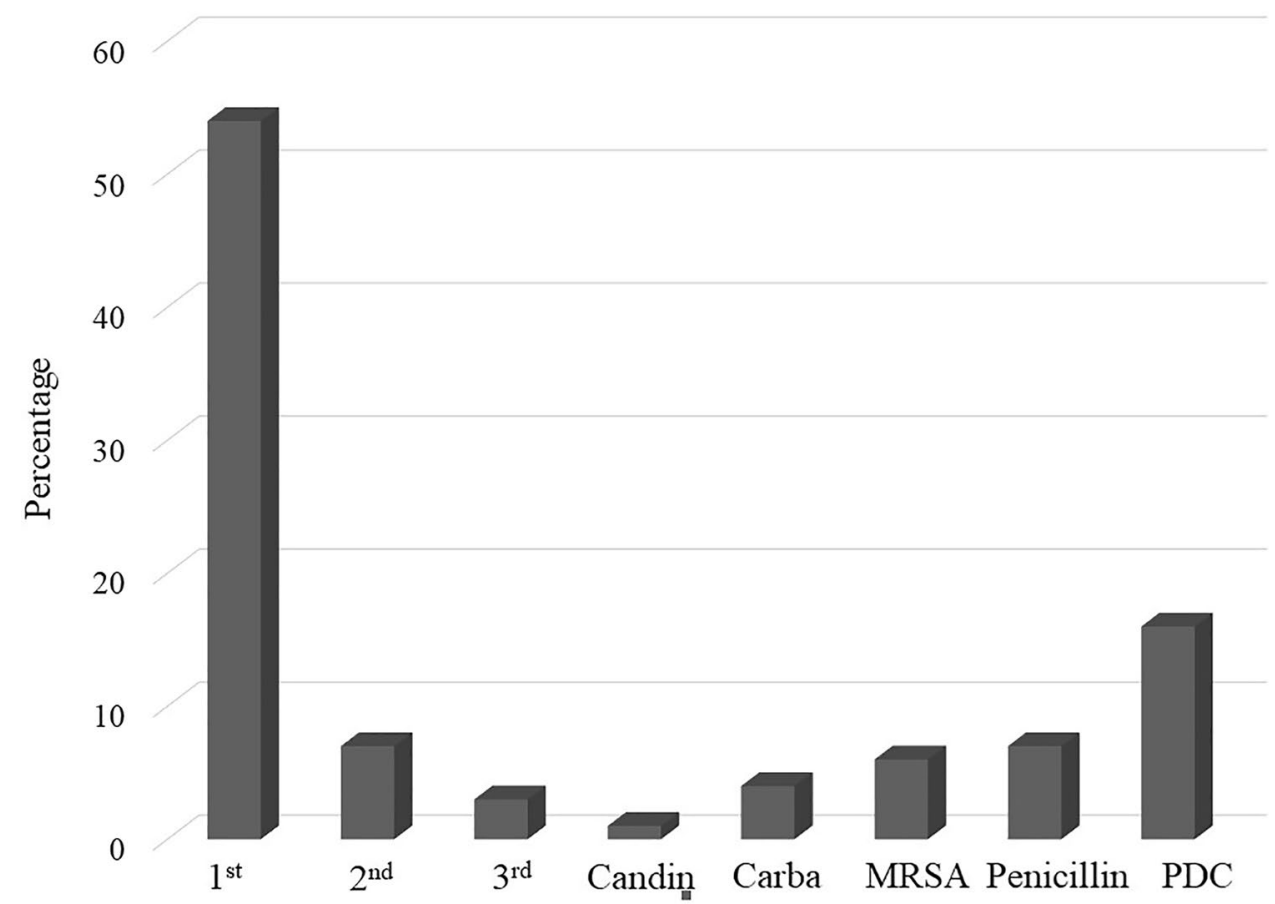


should consider the balance between individual and social risk/benefit ratios from the viewpoint of recent antimicrobial resistance patterns [6]. The 10-year lapse between the ELSO survey and our survey might affect the lower use of routine antimicrobial prophylaxis in Japan.

Recently, a single-center study showed that the protocol reduced the use of broad-spectrum antimicrobial agents such as cefepime, vancomycin, and carbapenem, while 30-day mortality and nosocomial infections did not change [7]. Another recent study evaluating the efficacy of prophylactic antibiotics using national data for 9615 ECMO patients from 2010 to 2017 in Japan revealed that 58\% of patients were administered prophylactic antibiotics, and hospital mortality and nosocomial pneumonia in the prophylaxis group were significantly lower than those in the control group (56\% vs. $60 \%$ and $13 \%$ vs. $15 \%$, respectively) [8]. The effectiveness of antimicrobial prophylaxis and the optimal methods during ECMO remain unclear because of a lack of large prospective studies on this subject. Our study revealed that duration of antimicrobial prophylaxis was not determined in $47 \%$ of hospitals. We believe that the lack of evidence and the absence of a standardized protocol might be a reason for this result.

In terms of routine infection surveillance, our survey indicated that the proportion of blood and sputum samples obtained for cultures in Japan were 72 and 86\%, respectively, while they were 100 and $48 \%$, respectively, in the ELSO survey. A previous international survey reported that infection surveillance differed according to geographical region [1]. In Japan, routine blood culture was not as popular as in other countries. Physicians might perform infection surveillance as needed based on their experience because of a lack of evidence regarding optimal surveillance $[1,3]$. Further high-quality studies are needed to eliminate regional and inter-hospital disparities.

This study has several limitations. First, we did not collect outcomes data. Thus, we could not draw a conclusion on whether antimicrobial prophylaxis and standardized protocols influenced outcomes. Second, the subjects of this survey were intensivists and hospitals belonging to the JSICM. Therefore, we did not include all hospitals that administer ECMO in Japan. In addition, different intensivists had unique opinions, even in the same hospital, because many hospitals did not have a standardized protocol.

\section{Conclusions}

Our survey revealed that $39 \%$ of hospitals administered antimicrobial prophylaxis during ECMO in Japan. In addition, first-generation cephalosporins were the agents most commonly used. However, only $17 \%$ of hospitals have a standardized protocol. Prospective multicenter observational studies about the relationship between antimicrobial prophylaxis and outcomes are required to create a guideline.

Supplementary Information The online version contains supplementary material available at https://doi.org/10.1007/s10047-021-01291-3.

\section{References}

1. Farrell D, MacLaren G, Schlapbach LJ. Infections on extracorporeal life support in adults and children-A survey of international practice on prevention, diagnosis, and treatment. Pediatr Crit Care Med. 2019;20:667-71.

2. O'Horo JC, Cawcutt KA, De Moraes AG, Sampathkumar P, Schears GJ. The evidence base for prophylactic antibiotics in patients receiving extracorporeal membrane oxygenation. ASAIO J. 2016;62:6-10.

3. Kao LS, Fleming GM, Escamilla RJ, Lew DF, Lally KP. Antimicrobial prophylaxis and infection surveillance in extracorporeal membrane oxygenation patients: a multi-institutional survey of practice patterns. ASAIO J. 2011;57:231-8.

4. Morris AM, Bai A, Burry L, Dresser LD, Ferguson ND, Lapinsky SE, Lazar NM, McIntyre M, Matelski J, Minnema B, Mok K, Nelson S, Poutanen SM, Singh JM, So M, Steinberg M, Bell CM. Long-term effects of phased implementation of antimicrobial stewardship in academic ICUs: 2007-2015. Crit Care Med. 2019;47:159-66.

5. Tonna JE, Abrams D, Brodie D, Greenwood JC, Rubio MateoSidron JA, Usman A, Fan E, Barrett N, Schmidt M, Mueller T, Combes A, Shekar K. Management of adult patients supported with venovenous extracorporeal membrane oxygenation (VV ECMO): guideline from the extracorporeal life support organization (ELSO). ASAIO J. 2021. https://doi.org/10.1097/mat.00000 00000001432.

6. Depuydt P, De Waele JJ. Optimal and responsible use of antibiotics. Curr Opin Critical Care. 2019;25:458-64.

7. Shah A, Sampathkumar P, Stevens RW, Bohman JK, Lahr BD, Dhungana P, Vashistha K, O'Horo JC. Reducing broad spectrum antimicrobial use in extracorporeal membrane oxygenation (ECMO): reduce AMMO study. Clin Infect Dis. 2021. https://doi. org/10.1093/cid/ciab118.

8. Kondo Y, Ohbe H, Aso S, Matsui H, Fushimi K, Tanaka H, Yasunaga $\mathrm{H}$. Efficacy of prophylactic antibiotics during extracorporeal membrane oxygenation: a nationwide cohort study. Ann Am Thorac Soc. 2021. https://doi.org/10.1513/AnnalsATS. 202008-974OC.

Publisher's Note Springer Nature remains neutral with regard to jurisdictional claims in published maps and institutional affiliations. 